\title{
Polarization switching in quantum-well vertical-cavity surface-emitting lasers
}

\author{
J. Martín-Regalado, M. San Miguel, and N. B. Abraham* \\ Departament de Física, Universitat de les Illes Balears and Instituto Mediterraneo de Estudios Avanzados, \\ IMEDEA (CSIC-UIB), E-07071 Palma de Mallorca, Spain \\ F. Prati \\ Department of Physics, University of Milan, via Celoria 16, 20133 Milan, Italy
}

Received August 7, 1995

\begin{abstract}
Switching between linearly polarized states of slightly different optical frequencies with the same transversemode pattern is found, as the injection current is increased. Switchings found here for semiconductor rateequation models incorporating a vector electric field, birefringence, and the $\alpha$ factor are similar to previously reported experimental results. (c) 1996 Optical Society of America
\end{abstract}

Changes in the polarization state of the emitted field are sometimes seen as the excitation is changed for vertical-cavity surface-emitting lasers (VCSEL'S). Sometimes higher-order transverse modes appear, ${ }^{1-4}$ while in other situations the polarization state changes while the mode pattern remains fixed. ${ }^{2-9}$ Emission is typically linearly polarized, oriented along the crystal axes. Birefringence of the crystal splits the emission frequencies of orthogonal linearly polarized fields ${ }^{8}$ (see also Refs. 1-3 and Ref. 5), and sometimes there is emission on both polarizations when a difference in the optical frequencies cannot be resolved. ${ }^{2-4,10}$ The only explanation offered for the selection of a particular polarization is that one has a more favorable gain-toloss ratio and that switchings arise from changes in the relative gain-to-loss ratios (see, e.g., Ref. 3).

An alternative explanation, explored in this Letter, is that polarization-state switching arises from the coupling of the vector field to the saturable dispersion (represented by the $\alpha$ factor in semiconductor rateequation models) when the material is birefringent.

A rate-equation version of a recently developed model $^{11}$ appropriate for VCSEL'S operating on a single longitudinal spatial mode with the lowest-order transverse field pattern is as follows:

$$
\begin{aligned}
\frac{\mathrm{d} E_{ \pm}}{\mathrm{d} t}= & -\kappa E_{ \pm}-i \omega_{0} E_{ \pm}+\kappa(1+i \alpha)(N \pm n) E_{ \pm} \\
& -i \gamma_{p} E_{\mp}-\gamma_{a} E_{\mp}, \\
\frac{\mathrm{d} N}{\mathrm{~d} t}= & -\gamma(N-\mu)-\gamma(N+n)\left|E_{+}\right|^{2}-\gamma(N-n)\left|E_{-}\right|^{2},
\end{aligned}
$$

$$
\frac{\mathrm{d} n}{\mathrm{~d} t}=-\gamma_{s} n-\gamma(N+n)\left|E_{+}\right|^{2}+\gamma(N-n)\left|E_{-}\right|^{2},
$$

where for convenience we have decomposed the slowly varying amplitude of the vector electric field into amplitudes $E_{ \pm}$of the left (-) and right (+) circularly polarized basis states. $N$ is the total population dif- ference between conduction and valence bands, and $n$ is the difference of the population differences between the two allowed transitions between magnetic sublevels. We recover the physics of the usual semiconductor laser rate equations by setting the variable $n$ to zero. $\quad \kappa$ is the field decay rate, $\alpha$ is the linewidth enhancement factor, $\omega_{0}=\kappa \alpha$ is a frequency shift that leads to a zero optical frequency for the slowly varying amplitude of the field at the lasing threshold in the absence of anisotropies, $\gamma_{p}$ gives the relative detuning of the linearly polarized modes, and $\gamma_{a}$ is the strength of the anisotropic losses for the linearly polarized modes. We can most clearly display the meaning of the parameters $\gamma_{p}$ and $\gamma_{\alpha}$ by rewriting Eq. (1) in the $x-y$ basis:

$$
\begin{aligned}
\frac{\mathrm{d} E_{ \pm}}{\mathrm{d} t}= & -\left(\kappa+\gamma_{a}\right) E_{x}-i\left(\omega_{0}+\gamma_{p}\right) E_{x} \\
& +\kappa(1+i \alpha)\left(N E_{x}+i n E_{y}\right), \\
\frac{\mathrm{d} E_{y}}{\mathrm{~d} t}= & -\left(\kappa-\gamma_{a}\right) E_{y}-i\left(\omega_{0}-\gamma_{p}\right) E_{y} \\
& +\kappa(1+i \alpha)\left(N E_{y}+i n E_{x}\right) .
\end{aligned}
$$

Other parameters in Eqs. (2) and (3) are the decay rate $\gamma$ of the total carrier population; the normalized injection current $\mu$, which takes the value 1 at the lasing threshold; and the decay rate $\gamma_{s}$, which accounts for the elimination of magnetic sublevel population differences through spin-flip relaxation processes, ${ }^{11,12}$ occurring on the order of tens of picoseconds. ${ }^{13}$ The fact that the dynamics of the sublevel populations cannot be neglected through an adiabatic elimination of fast processes is evident when one considers that the decay rate $\gamma_{s}$ is of the order of the time dependence (beating) resulting from the frequency splitting caused by $\gamma_{p}$ and is less than the field decay rate. Previous analyses of gas lasers found that polarization-state dynamics depended sensitively on the frequency splitting of the modes, the loss anisotropies of the medium, and contributions of the material dynamics to the cross saturation of the orthogonally polarized fields. ${ }^{14}$ VCSEL'S have the first two characteristics naturally from crystal properties and from 
geometrical distortions, and the third is contained in the spin-sensitive dynamical model for the carriers.

Without anisotropies, any linearly polarized field is a solution of Eqs. (1) - (3), and dynamically the orientation diffuses because of noise. However, the presence of birefringence $\left(\gamma_{p} \neq 0\right)$ fixes the polarization emission axes and gives the $\hat{x}$ - and $\hat{y}$-polarized solutions frequency shifts of $\gamma_{p}$ and $-\gamma_{p}$, respectively.

In narrow parameter regions we have also found simultaneous emission of differently polarized fields at different wavelengths as well as elliptically polarized solutions, phenomena that correspond to certain experimental observations (Refs. 2-4 and Ref. 15, respectively). Vigorous pulsations of the intensities of the polarized components are sometimes observed, with characteristic frequencies corresponding to the frequency splitting of the steady-state solutions $(2-20 \mathrm{GHz}$, experimentally).

The linear stability of the linearly polarized states is determined by the following characteristic polynomial:

$$
\begin{aligned}
P(\lambda)= & \lambda^{3}+\left(\frac{\gamma_{s}}{\gamma}+\mu-1\right) \lambda^{2} \\
& +\left[2 \frac{\kappa}{\gamma}(\mu-1)+4\left(\frac{\gamma_{p}}{\gamma}\right)^{2}\right] \lambda \mp 4 \frac{\kappa \gamma_{p}}{\gamma^{2}} \\
& \times \alpha(\mu-1)+4\left(\frac{\gamma_{p}}{\gamma}\right)^{2}\left(\frac{\gamma_{s}}{\gamma}+\mu-1\right),
\end{aligned}
$$

where $\mp$ applies to the $\hat{x}$-polarized and the $\hat{y}$-polarized solutions, respectively. The stability boundaries for the linearly polarized solutions in the phase space $\left(\mu\right.$ versus $\left.\gamma_{p} / \gamma\right)$ are represented in Fig. 1 . The $\hat{x}$ polarized solution is stable when

$$
\left(\frac{\gamma_{p}}{\gamma}\right)_{x}>\left[\frac{\kappa}{\gamma} \alpha(\mu-1)\right] /\left(\frac{\gamma_{s}}{\gamma}+\mu-1\right)
$$

(below the solid curve), and the $\hat{y}$-polarized solution is stable when

$$
\left(\frac{\gamma_{p}}{\gamma}\right)_{y}<\left(\frac{\gamma_{s}}{\gamma}+\mu-1\right) / 2 \alpha
$$

(to the left of the dashed line). What makes a crucial difference in their stabilities is the relative detuning of the linearly polarized solutions with respect to the sign of $\alpha$.

To demonstrate some of the types of switching possible within this model, we have varied the injection current for $\gamma_{p}=2 \gamma$ and $\gamma_{p}=10 \gamma$. For comparison with typical experimental results we show first the time-averaged power of the $\hat{x}$ - and $\hat{y}$-polarized components. For $\gamma_{p}=2 \gamma$ there are two possible results since just above threshold $(\mu=1)$ both solutions are stable: (i) if the system begins with the $\hat{y}$-polarized solution, it retains this polarization as $\mu$ is raised and lowered; (ii) if the system begins with $\hat{x}$-polarized emission, it switches to $\hat{y}$-polarized emission after passing successively through elliptically polarized emission, and more complex time-dependent emission (modulated at the optical beat frequency of order $\gamma_{p}$ ). We show this transition in Fig. 2(a). In this case, if $\mu$ is raised enough to produce $\hat{y}$-polarized emission, this polarization state is retained stably when $\mu$ is lowered, yielding an evident hysteresis signature. Spontaneous emission noise and the relative stability and spectral response of the two stable states lead to the selection of the initial polarization state, as the current is raised above threshold. We have not found

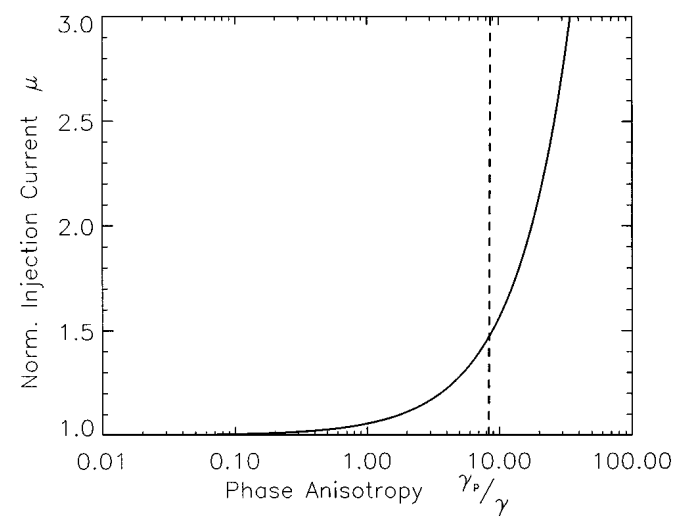

Fig. 1. Stability diagram of the linearly polarized solutions for the parameters $\kappa=300 \mathrm{~ns}^{-1}, \gamma=1 \mathrm{~ns}^{-1}, \gamma_{s}=$ $50 \mathrm{~ns}^{-1}$, and $\alpha=3$.
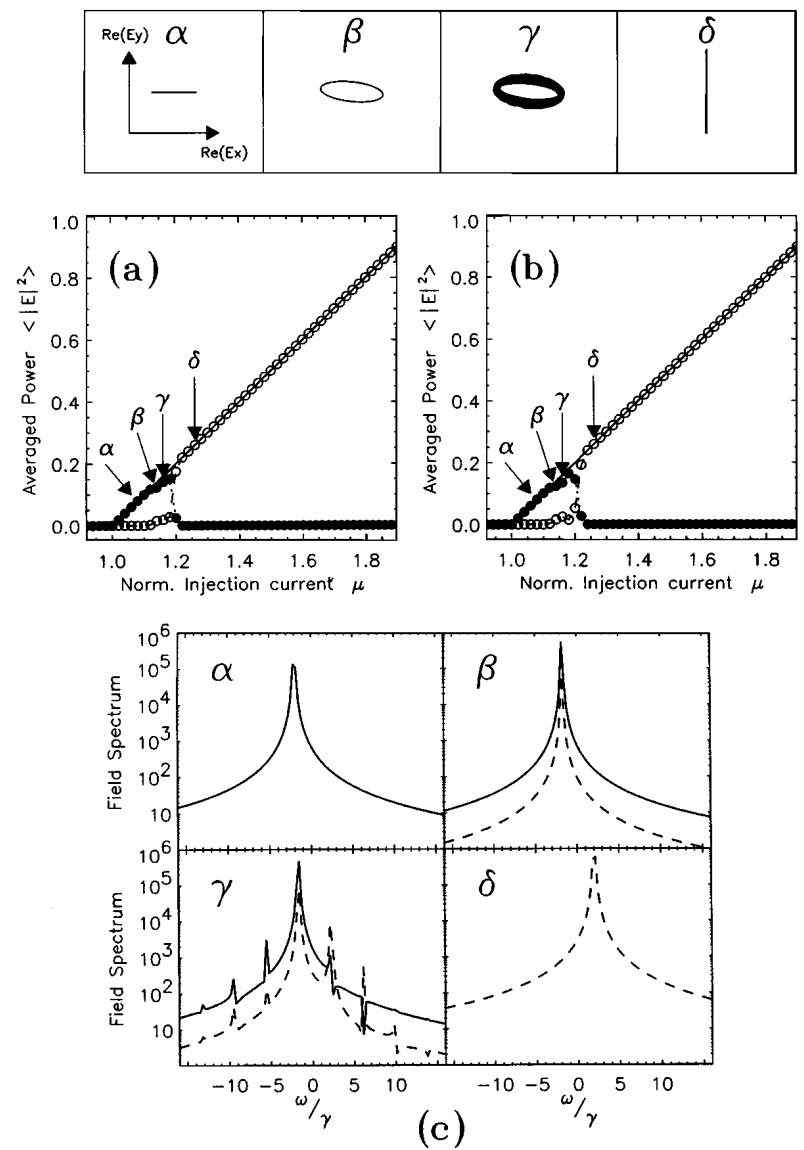

Fig. 2. Time-averaged (20-ns) power of the $\hat{x}$-polarized (filled circles) and $\hat{y}$-polarized (open circles) field components and their total (solid lines) for $\gamma_{p}=2 \gamma$, showing switching in (a) the absence $\left(\gamma_{a}=0\right)$ and (b) the presence $\left(\gamma_{a}=-0.1 \gamma\right)$ of linear amplitude anisotropy. For selected (indicated) currents, $\operatorname{Re}\left(E_{\gamma}\right)$ versus $\operatorname{Re}\left(E_{x}\right)$ is shown at the top of the figure, and the optical spectra are shown in (c), in which $\hat{x}$ - and $\hat{y}$-polarized field spectra are indicated by solid and dashed curves, respectively. 

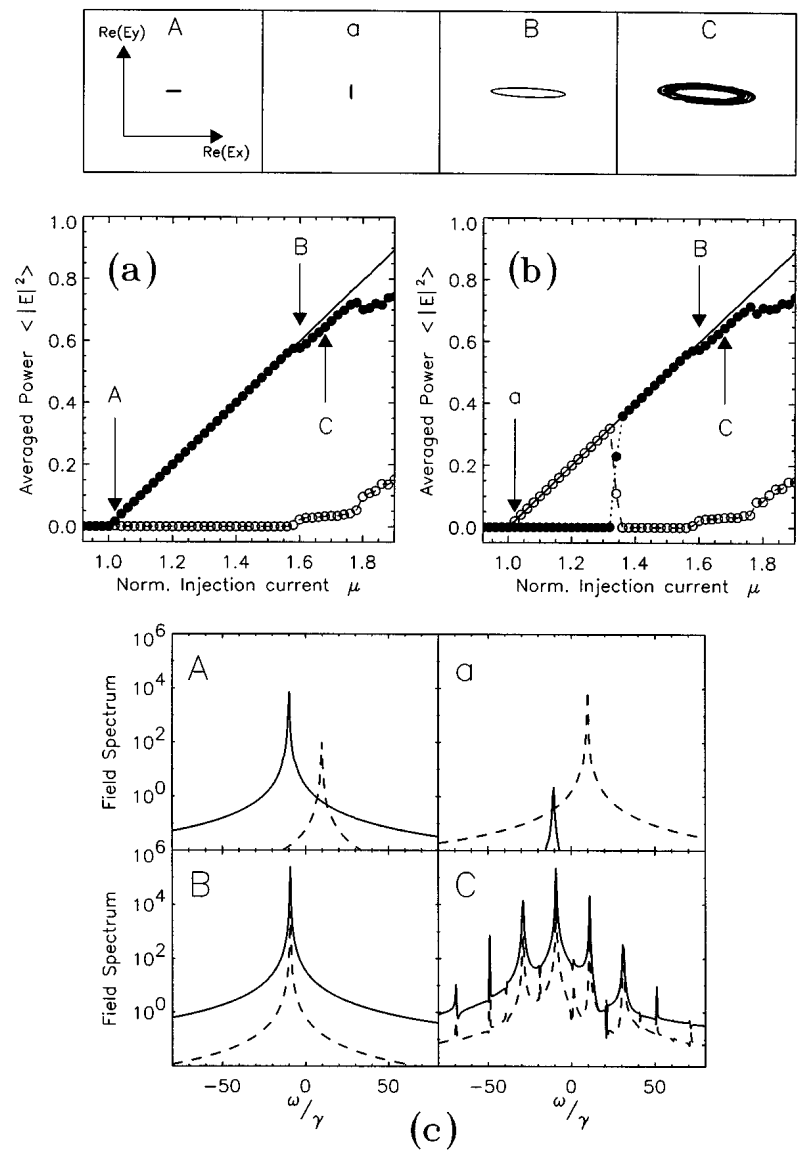

Fig. 3. Time-averaged power for $\gamma_{p}=10 \gamma$ in (a) the absence $\left(\gamma_{a}=0\right)$ and (b) the presence $\left(\gamma_{a}=0.1 \gamma\right)$ of linear amplitude anisotropy. Other details as in Fig. 2.

experimental reports of such nonreproducible initial polarization-state selection as the current is raised or lowered with hysteresis in one case.

However, near this value of $\gamma_{p}$ unique switching transitions occur that are similar to those found experimentally, if there is a small loss anisotropy favoring the $\hat{x}$-polarized state $\left(\gamma_{a}<0\right)$, as shown in Fig. 2(b). This is similar to Fig. 2(a), but recall that Fig. 2(a) was one of two possible results when $\gamma_{a}=0$. The associated polarization of $\operatorname{Re}(\mathbf{E})$ for certain current values indicated with arrows is shown in the top of the figure. Figure 2(c) shows the optical spectra at these points. The $\hat{x}$ - and $\hat{y}$-polarized solutions have different optical frequencies. Elliptically polarized emission and states of modulated polarization appear in a narrow transition zone.

For sufficiently large frequency splittings $\left(\gamma_{p}=10 \gamma\right)$ in the absence of loss anisotropy there is no abrupt switching at low values of the injection current, as shown in Fig. 3(a). The lethargy of the spin dynamics leads to the selection of the $\hat{x}$-polarized solution (because of the signs of $\alpha$ and $\gamma_{p}$ ) rather than permitting both solutions near threshold. The saturation of the intensity of the $\hat{x}$-polarized state observed at larger values of the injection current and the onset of the $\hat{y}$-polarized field appear similar to experimental results (Ref. 2, among others), but those results are clearly explained by the appearance of transverse modes, a phenomenon not included here. However, an abrupt switching in the polarization of the fundamental spatial mode such as that observed experimentally occurs in our model in this parameter range when we include a small loss anisotropy favoring $\hat{y}$-polarized emission $\left(\gamma_{a}>0\right)$. This is shown in Fig. 3(b), in which the behavior for values of $\mu$ above the abrupt switch is nearly the same as in Fig. 3(a).

The results demonstrate the effects of the conventional $\alpha$ factor of semiconductor rate equations when birefringence and a weak loss anisotropy are included. Figure 3 reveals additional and somewhat different phenomena resulting from a finite spin relaxation rate. Although we have not included changes in the relative gains of the modes as the current is increased, the fact that saturable dispersion effects may select emission on the polarization state with the lesser gain-to-loss ratio suggests that the effects incorporated in our model may be more important than (or as important as) the gain-to-loss differential, at least when the gain differential is small (when the frequency splittings are small compared with the width of the gain profiles).

This study was partially supported by CICYT (Spain) projects TIC95/0563 and PB94/1167 and European Union HCM grant CHRX-CT-93-0114.

*Permanent address, Department of Physics, Bryn Mawr College, 101 North Merion Avenue, Bryn Mawr, Pennsylvania 19010-2899.

\section{References}

1. C. J. Chang-Hasnain, J. P. Harbison, G. Hasnain, A. C. Von Lehmen, L. T. Florez, and N. G. Stoffel, IEEE J. Quantum Electron. 27, 1402 (1991).

2. K. D. Choquette and R. E. Leibenguth, IEEE Photon. Technol. Lett. 6, 40 (1994).

3. K. D. Choquette, D. A. Richie, and R. E. Leibenguth, Appl. Phys. Lett. 64, 2062 (1994).

4. H. Li, T. L. Lucas, J. G. McInerney, and R. A. Morgan, Chaos Solitons Fractals 4, 1619 (1994).

5. Z. G. Pan, S. Jiang, and M. Degenais, Appl. Phys. Lett. 63, 2999 (1993).

6. A. Chavez-Pirson, H. Ando, H. Saito, and H. Kanbe, Appl. Phys. Lett. 62, 3082 (1993).

7. K. D. Choquette, K. L. Lear, R. E. Leibenguth, and M. T. Asom, Appl. Phys. Lett. 64, 2767 (1994).

8. A. K. Jansen van Doorn, M. P. van Exter, and J. P. Woerdman, Electron. Lett. 30, 1941 (1994).

9. S. Jiang, Z. Pan, M. Dagenais, R. A. Morgan, and K. Kojima, Appl. Phys. Lett. 63, 3545 (1993).

10. C. J. Chang-Hasnain, J. P. Harbison, L. T. Florez, and N. G. Stoffel, Electron. Lett. 27, 163 (1991).

11. M. San Miguel, Q. Feng, and J. V. Moloney, Phys. Rev. A 52, 1728 (1995).

12. F. Meier and B. P. Zachachrenya, eds., Optical Orientation (North-Holland, Amsterdam, 1984).

13. T. C. Damen, L. Vina, J. E. Cunningham, J. Shah, and L. J. Sham, Phys. Rev. Lett. 67, 3432 (1991); S. Bar-Ad and I. Bar-Joseph, Phys. Rev. Lett. 68, 349 (1992).

14. W. Van Haeringen, Phys. Rev. 158, 256 (1967); D. Lenstra, Phys. Rep. 59, 299 (1980).

15. M. P. van Exter, A. K. Jansen van Doorn, R. F. M. Hendriks, and J. P. Woerdman, Proc. SPIE 2693 (1996); A. K. Jansen van Doorn, M. P. van Exter, M. Travagnin, and J. P. Woerdman (personal communication, 1996). 